\title{
Zwischen Datenhunger und Nachhaltigkeit
}

\begin{abstract}
Digitalisierung und Nachhaltigkeit - wie kann das gelingen? Ein Kernelement ist dabei die Verarbeitung der stetig zunehmenden Datenmengen und der damit zusammenhängende Betrieb von Rechenzentren. Dr. Doris Dobida, Expertin der staatlichen Betriebsniederlassungsagentur Invest in Austria, erklärt im Interview die Trends in diesem Bereich. Interview mit Doris Dobida
\end{abstract}

$S^{i}$ ie gehören zu den größten Herausforderungen unserer Zeit: Das Verarbeiten endloser Datenmengen auf der einen und der Kampf gegen den Klimawandel auf der anderen Seite. Angesichts des gigantischen Energiebedarfs des ersten scheinen diese beiden Ziele unvereinbar. Green Datacenter, also möglichst klimaneutrale Rechenzentren, versprechen eine Lösung dieses modernen Dilemmas. Wichtige Fragen sind, welcher Rahmenbedingungen es bedarf, um solche Center erfolgreich zu machen, und wie gezielte Förderprogramme für die richtigen Anreize bei Unternehmen und Investoren sorgen.

ÖW: Wie wichtig sind Green Datacenter und welchen Beitrag können sie zu einer umweltbewussten und zugleich digitalisierten Zukunft leisten?

Dobida: Rechenzentren im Allgemeinen bilden das Herz des gigantischen Datenkreislaufs, der unsere moderne, digitale Welt erst ermöglicht. Jegliche Information muss hier verarbeitet werden und genau wie das menschliche Herz muss auch das Rechenzentrum nonstop in Betrieb sein. Ausfälle würden zu immensen wirtschaftlichen Schäden führen und wären somit unverzeihlich.

Angesichts der stetig fortschreitenden Digitalisierung unseres Alltags- und Geschäftslebens wird die Menge an zu verarbeitenden Daten und mit ihr der Bedarf an effizienten Rechenzentren auch weiterhin wachsen.
Das große Manko von Rechenzentren ist allerdings ihr enormer Energieverbrauch. Bei einer Nutzung herkömmlicher, fossiler Energieressourcen ist dieser mit den Klimazielen aus dem Pariser Abkommen unvereinbar. Hier kommen Green Datacenter, also Rechenzentren, die zu großen Teilen aus erneuerbaren Energien versorgt werden, ins Spiel. Sie stellen das Bindeglied zwischen einem gesteigerten Bedarf an Rechenleistung und ökologischem Bewusstsein dar.

Welche Voraussetzungen bezüglich der Infrastruktur müssen erfüllt sein, um moderne Rechenzentren wirklich mit Strom aus erneuerbaren Energien zu versorgen?

Damit ein Green Datacenter den Spagat zwischen hoher Leistungsfähigkeit im Dauerbetrieb und Nachhaltigkeit meistern kann, ist die richtige Infrastruktur absolut entscheidend.

An erster Stelle steht selbstverständlich die Gewährleistung einer lückenlosen Energieversorgung. Dabei kommt es auf einen intelligenten Mix aus verschiedenen Energiequellen an. Allerdings sind auch hier manche Quellen attraktiver als andere.

In Österreich zum Beispiel liegt der Anteil erneuerbarer Energien an der gesamten Stromproduktion bei knapp $80 \%$. Dabei setzt man vor allem auf Wasserkraft. Diese sorgt nicht nur für „grünen“ Strom, sondern bietet größtmögliche Versorgungssicherheit.
Zugleich ist Wasser auch die Lösung für eine der größten Herausforderungen, wenn es darum geht, den erforderlichen Dauerbetrieb zu gewährleisten: die Kühlung der einzelnen Rechner. Nur um an dieser Stelle zu veranschaulichen, wie hoch der Kosten- und Energieaufwand für eine entsprechende Kühlung ist. Bei Temperaturen jenseits der dreißig Grad Celsius, wie sie besonders in den letzten Sommern in Europa keine Seltenheit waren, kostet es entsprechend Geld.

Ließe sich dieses Problem mit der Verlagerung des Datacenters an einen kühleren Standort, zum Beispiel nach Skandinavien, lösen?

Wenn man wirklich von sowohl wirtschaftlich rentablen als auch nachhaltigen Datacenters sprechen möchte, müssen diese auch an modernste Glasfasernetze angebunden sein. Denn es ist alles andere als effizient, wenn die verarbeiteten Daten dann wieder kilometerweit reisen müssen, bis sie an einem der wichtigen Knotenpunkte, zum Beispiel in der Mitte Europas, ankommen.

Gibt es noch weitere Aspekte, die darüber entscheiden, wie nachhaltig solch ein Green Datacenter wirklich ist?

Genau wie herkömmliche Rechenzentren haben auch Green Datacenter den ökologischen Nachteil, dass bei deren Bau große Flächen an Land versiegelt werden. Dies kommt auch bei den benachbarten Gemeinden nicht gut an. Neben dem ökologischen gibt es hier also auch ein Image-Problem für die Betreiber. Entsprechend bemüht ist man, das eigene Profil in Sachen Nachhaltigkeit zu schärfen. Manche Betreiber gehen dabei so weit, dass sie eigene Windkraft- oder Photovoltaik-Anlagen auf dem Gelände des Rechenzentrums anlegen. Dies ist aber nicht unbedingt notwendig, da mit der richtigen Infrastruktur nachhaltiger Strom auch aus dem herkömmlichen Netz bezogen werden kann. Alternativ werden gerne auch Partnerschaften oder Beteiligungen an Projekten zur grünen Stromgewinnung in der Region eingegangen. Durch diese gemeinnützigen Maßnahmen lässt sich nicht nur der öko- 
logische Nachteil der Versiegelung kompensieren. Auch das Verhältnis zu den Bewohnern der Region wird gestärkt.

Wie kann die Politik eine nachhaltige Digitalisierung für Unternehmen attraktiver gestalten? Sind gezielte Fördermaßnahmen aus Ihrer Sicht der richtige Weg?

Um die digitale Revolution auch nachhaltig zu gestalten, bedarf es aus der Politik in jedem Fall Anreize für Unternehmen. Hierbei kommt es darauf an, Standortvorteile zu nutzen. In Österreich gibt es deshalb eine Reihe an Gesetzen und Förderinitiativen, insbesondere für Unternehmen aus den Bereichen Energie und Datenverkehr. Schon 2012 hat Österreich mit einem eigenen Ökostromgesetz neue Ausbauziele für die Energieträger Wasserkraft, Windkraft, Biomasse/ Biogas und Photovoltaik festgelegt.

Aber auch modernste Anlagen der Kälteversorgungstechnik und der freien Kühlung sorgen für eine Optimierung der Energieeffizienz im Datencenter. So nutzt zum Beispiel das deutsche Rechenzentrum e-shelter in Wien nicht nur Strom aus erneuerbaren Energien, sondern auch die Abwärme wird für die Beheizung der Büroflächen und benachbarter Gebäude genutzt. Hierbei waren die Wiener Stadtwerke, Wien Energie und Wiener Netze ein wichtiger Umsetzungspartner auf kommunaler Ebene.

Abgesehen von politischen und technologischen Anreizen gewinnt das Thema Nachhaltigkeit auch aus ganz anderen Gründen bei Unternehmen an Attraktivität. Verbraucher/innen und Investoren legen immer mehr Wert auf das Thema, sodass sich ein grüner Ansatz auch aus wirtschaftlicher Sicht immer mehr lohnt. Nur wenn diese Entwicklung weiter vorangetrieben wird, kann die Trendwende gelingen.

AUTORIN + KONTAKT

Dr. Doris Dobida leitet den Fachbereich The Americas, Corporate Development bei ABA - Invest in Austria.

ABA Invest in Austria, Opernring 3, A-1010 Wien, Österreich. Tel.: +43 1885812 , E-Mail:d.dobida@aba.gv.at, Website: https://investinaustria.at/de/

\section{Briefe zur Inter disziplinarität}

Geistes-, Natur-,

Ingenieur- und

Sozialwissen-

schaften, Kunst

\& Handwerk

im Dialog
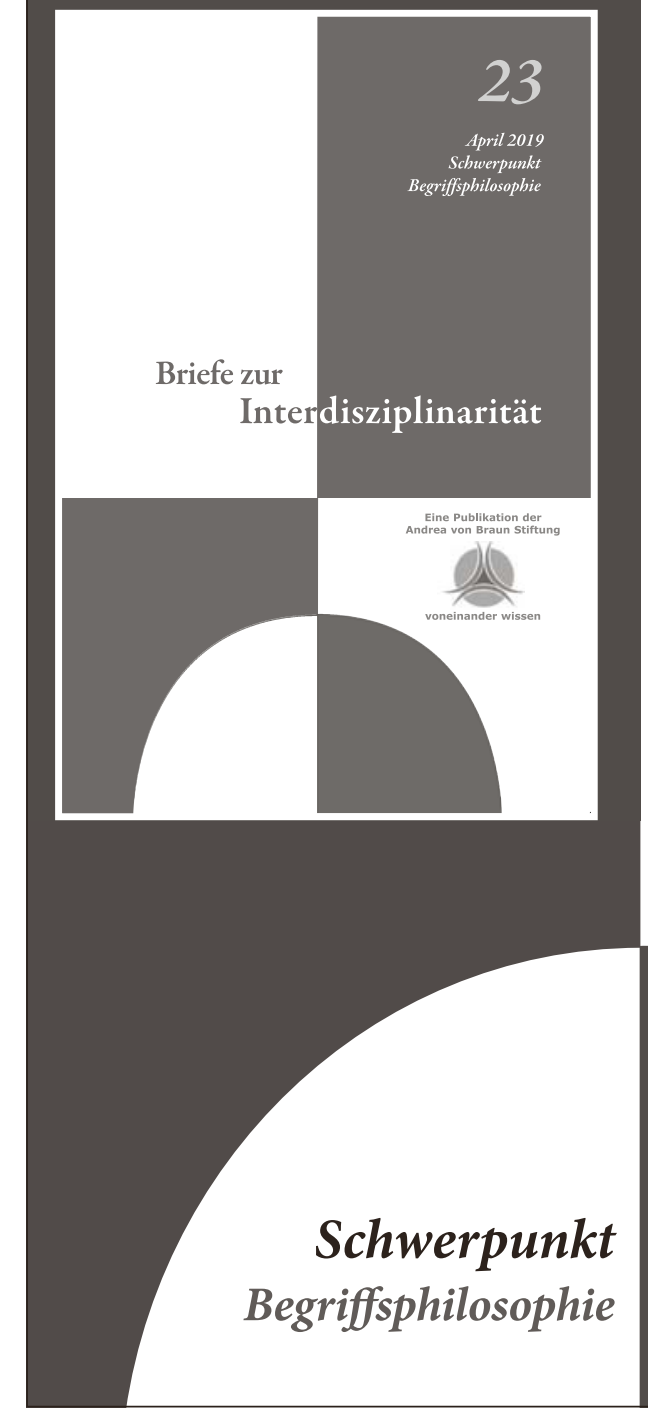

im oekom verlag, München. Mehr Informationen unter www.oekom.de/ briefe-zurInterdisziplinaritaet

Briefe zur Interdisziplinarität ist eine Publikation der Andrea von Braun Stiftung, Düsseldorf. Mehr Informationen zur Stiftung und ihren Zielen finden Sie unter www.avbstiftung.de

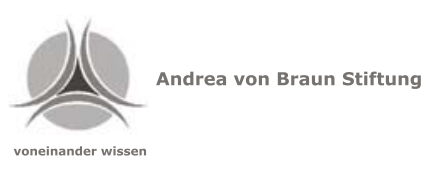

\section{/III oekom}

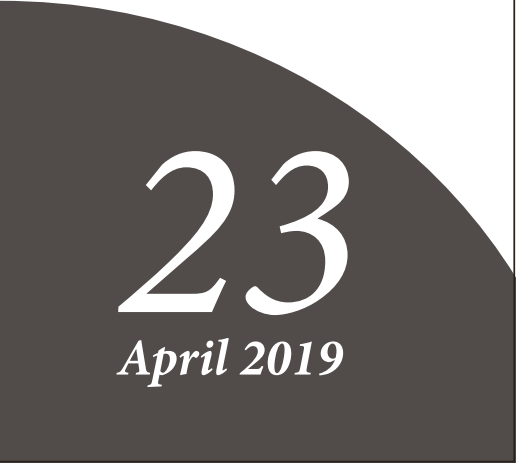

